\title{
A Case of Primary Central Nervous System Vasculitis That Worsened Despite Early Corticosteroid Therapy
}

\author{
Sang-Youl Yoon ${ }^{1}$, Ki-Su Park ${ }^{1}$, Seong-Hyun Park', Ji-Young Park ${ }^{2}$ \\ 'Department of Neurosurgery, Kyungpook National University School of Medicine, Kyungpook National University Hospital, Daegu, Korea \\ 2Department of Pathology, Kyungpook National University School of Medicine, Kyungpook National University Chilgok Hospital, Daegu, Korea
}

\author{
Received March 24, 2019 \\ Revised July 1, 2019 \\ Accepted July 3, 2019

\section{Correspondence} \\ Ki-Su Park \\ Department of Neurosurgery, \\ Kyungpook National University \\ School of Medicine, \\ Kyungpook National University Hospital, \\ 130 Dongdeok-ro, Jung-gu, \\ Daegu 41944, Korea \\ Tel: +82-53-200-5647 \\ Fax: +82-53-423-0504 \\ E-mail: kiss798@gmail.com
}

\begin{abstract}
Primary central nervous system vasculitis (PCNSV) is rare, and the diagnosis is difficult to make because of its variable radiologic expressions. Early corticosteroid therapy often is effective. Herein we report the case of a 56-year-old man who had a well-enhanced cystic mass with severe edema in the right frontal lobe, which was initially felt to be a malignancy. Histologic examination of tissue removed at craniotomy revealed that it was a PCNSV. Despite early administration of corticosteroids, a new lesion developed within 3 days. The lesions responded to treatment with cyclophosphamide and corticosteroid.
\end{abstract}

Key Words Vasculitis; Cysts; Neoplasms; Glucocorticoids.

\section{INTRODUCTION}

Primary central nervous system vasculitis (PCNSV) is rare, with a reported annual incidence of 2.4 cases per million [1]. Although the cause of this disorder is unknown, the pathogenesis is related to the inflammation of parenchymal and leptomeningeal arteries and veins [2]. The clinical manifestations are variable, and the disease usually is aggressive or fatal $[2,3]$. Making the diagnosis from radiologic findings may be difficult because of its variable patterns of expression [3]. Therefore, most cases are confirmed by histologic examination after excluding other diseases. Early corticosteroid or immunosuppressant treatment may result in a favorable outcome [2-4]. However, we report here a case of PCNSV mimicking a large malignant cystic tumor that worsened despite early corticosteroid therapy. The Institutional Review Board (IRB) of our hospital waived the approval of this study.

This is an Open Access article distributed under the terms of the Creative Commons Attribution Non-Commercial License (https://creativecommons.org/licenses/by-nc/4.0) which permits unrestricted non-commercial use, distribution, and reproduction in any medium, provided the original work is properly cited.

Copyright $\odot 2019$ The Korean Brain Tumor Society, The Korean Society for NeuroOncology, and The Korean Society for Pediatric Neuro-Oncology

\section{CASE REPORT}

A 56-year-old man was admitted to the hospital with mild headache after incurring a mild head trauma 3 days previously. He had a history of a gastrointestinal stromal tumor without hypertension, diabetes mellitus, or other manifestations. Brain CT revealed a low-density lesion in the right frontal lobe (Fig. 1A). MRI revealed a 6-cm cystic mass with low signal intensity on the T1-weighted image (WI) (Fig. 1B), a fluid-fluid level with upper high and lower low signal intensity on the T2-WI (Fig. 1C), and ring enhancement on the gadolinium contrast image (Fig. 1D). Diffusion WI revealed no restriction (Fig. 1E), but there was severe perilesional edema and mild subfalcial herniation. The provisional diagnosis was metastatic brain tumor or glioblastoma. High-dose corticosteroid (dexamethasone $30 \mathrm{mg}$ /day) was immediately administered, and MR spectroscopy was scheduled. However, mental deterioration from alert state to stupor and left hemiplegia developed on the 3rd day after admission. Brain CT revealed an increase in peritumor edema and subfalcial herniation (Fig. 2). Emergency craniotomy and total resection of the lesion, which consisted of a dense vascular capsule wall and 

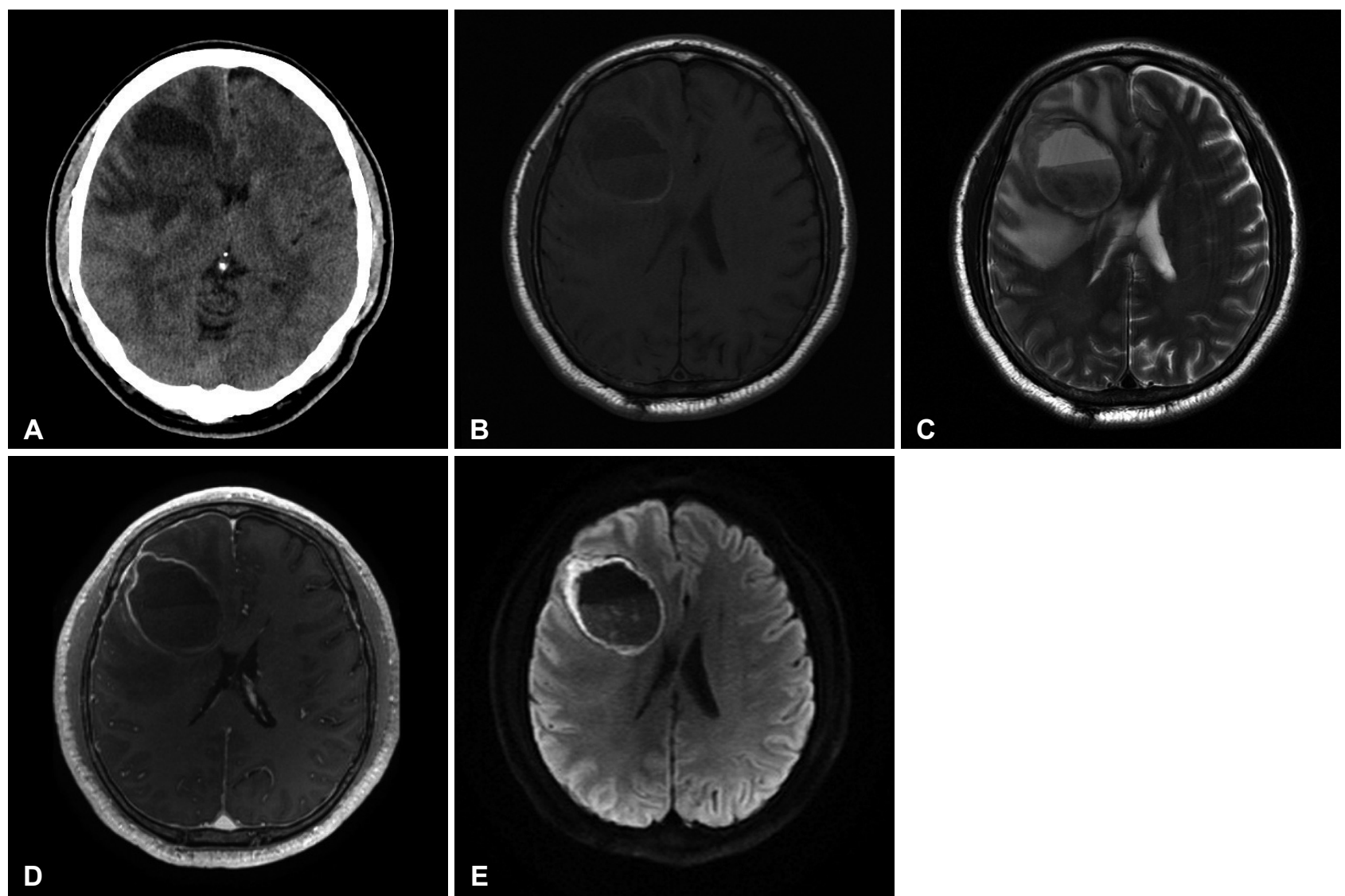

Fig. 1. Brain CT and MRI revealing a cystic mass-like lesion in the right frontal lobe. A: Brain CT reveals a 6-cm low-density lesion. B: Brain MRI illustrates the lesion with low signal intensity on T1-weighted image (WI). C: Fluid-fluid level of the lesion with upper high and lower low signal intensity is illustrated on T2-WI. D: Well-develop capsule is illustrated on enhanced MRI. E: Diffusion WI reveals no restriction.

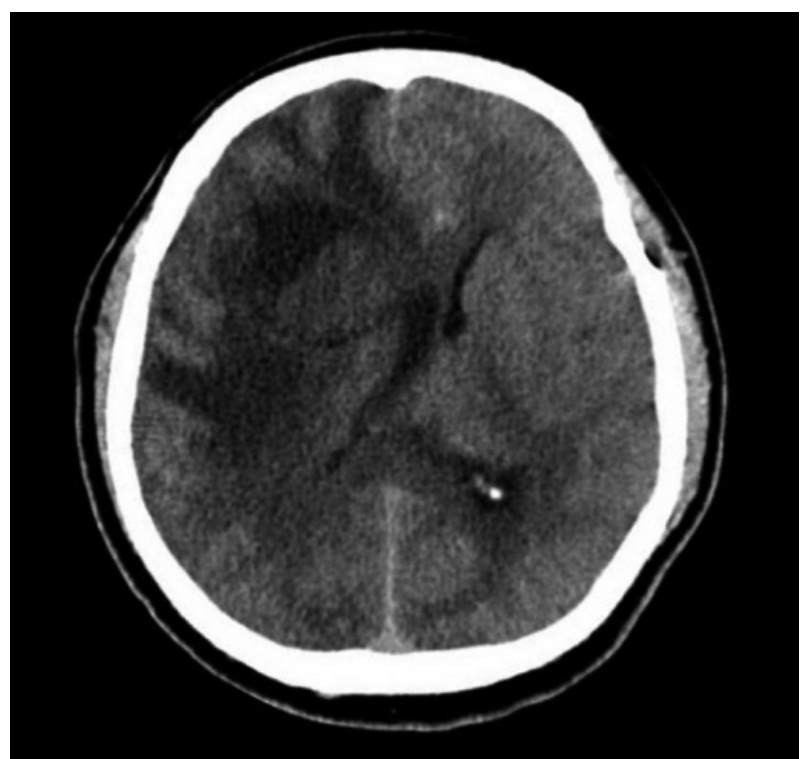

Fig. 2. Brain CT on 3rd day after admission illustrating increased peritumoral edema and subfalcial herniation.

cystic fluid with degenerated material, was performed.

Postoperatively, the patient regained consciousness but was drowsy. An immediate postoperative MRI revealed no resid- ual tumor at the primary site, but a new lesion with enhancement surrounding the occipital horn and trigone of the left lateral ventricle was evident (Fig. 3). Histopathologic examination revealed a pseudocapsule with prominent infiltration of CD3- and CD31-positive lymphocytes into small vessels (Fig. 4). These findings suggested the possibility of vasculitis. For differential diagnosis between vasculitis and other diseases mimicking vasculitis, including infections, autoimmune diseases, and lympho-proliferative disorders, laboratory tests on blood and CSF and gene rearrangement studies were performed. However, the C-reactive protein was only elevated to $3.11 \mathrm{mg} / \mathrm{dL}$. Additionally, the extent of vasculitis was assessed, and there was no lesion except brain lesions. Eventually, the PCNSV was confirmed. Combined cyclophosphamide and corticosteroid therapy was administered. Three months after surgery, brain MRI demonstrated complete remission.

\section{DISCUSSION}

In the management of central nervous system (CNS) tumors and tumor-like lesions, accurate diagnosis based on radiologic findings is essential for planning treatment and esti- 

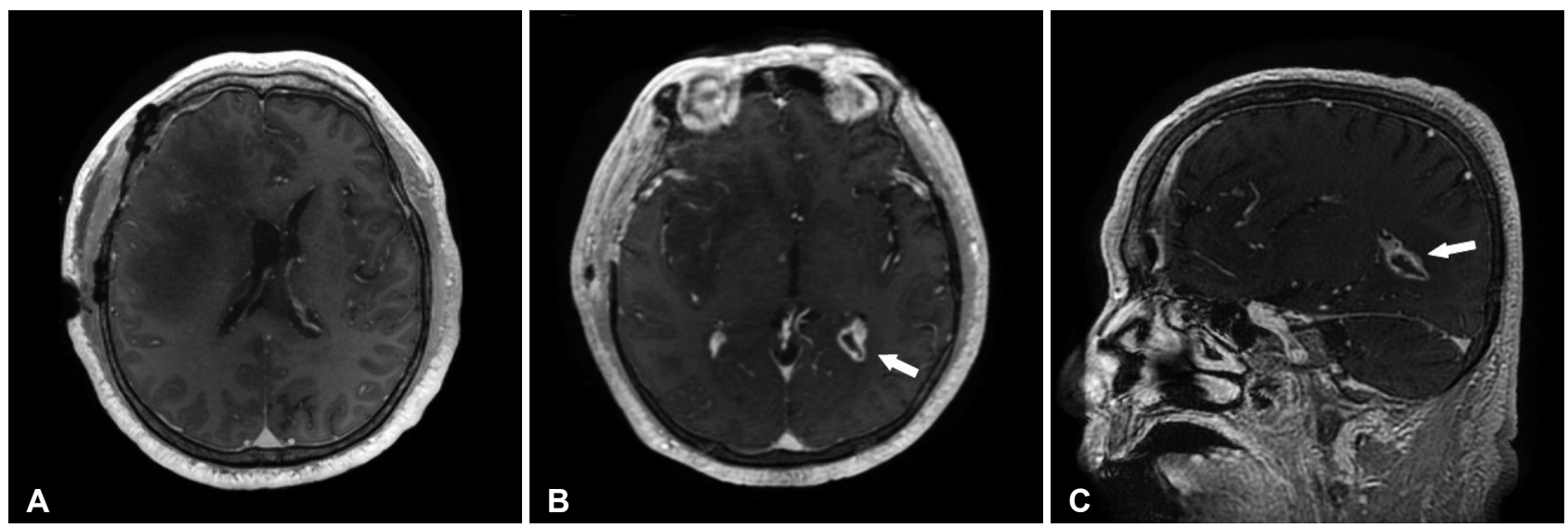

Fig. 3. Immediate postoperative MRI demonstrates no residual tumor at the primary site (A), but there was a new lesion with well enhancement surrounding the occipital horn and trigone of the left lateral ventricle (arrow) (B and C).
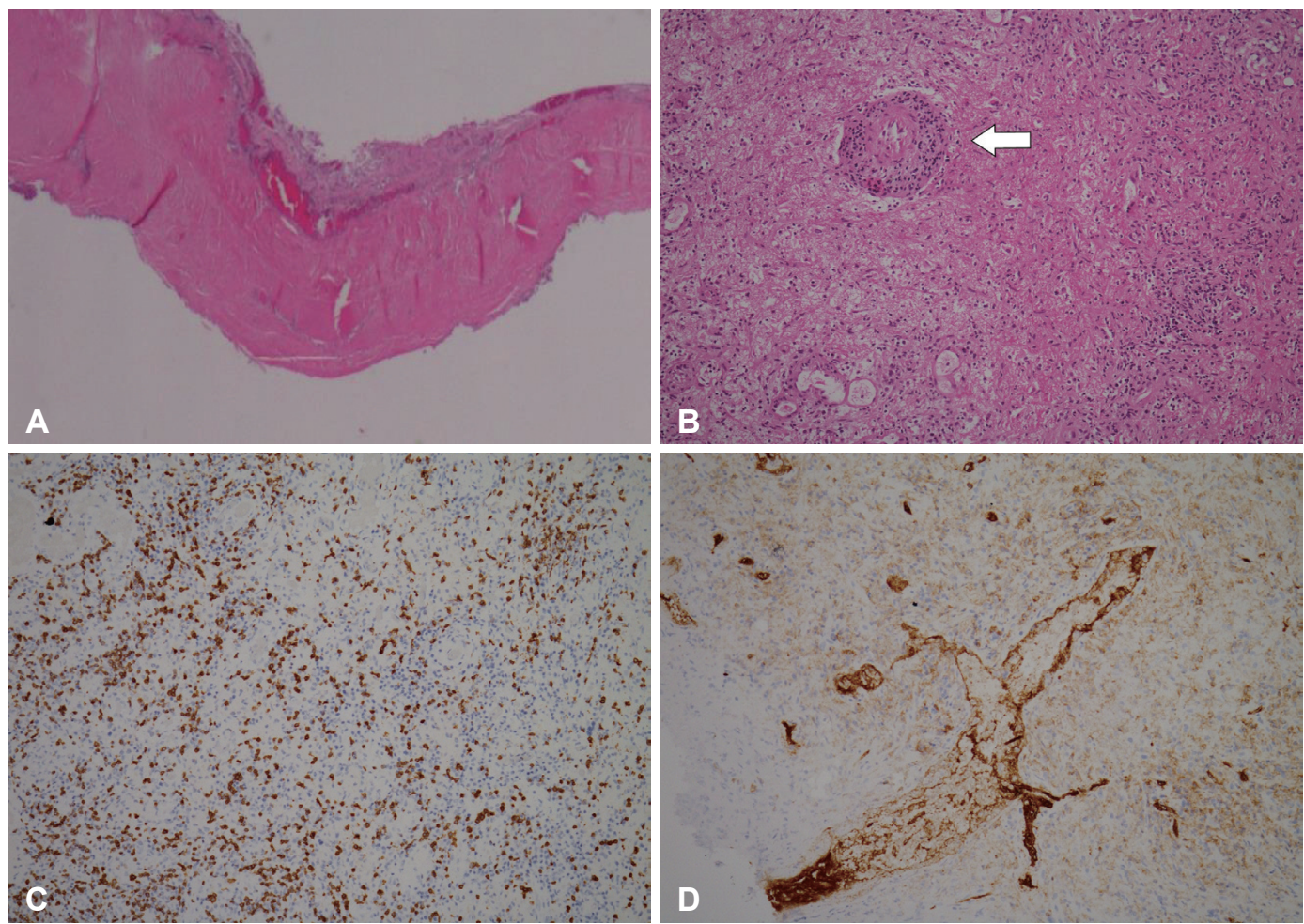

Fig. 4. Histological findings of vasculitis in the brain lesion. The hematoxylin-eosin stain demonstrated pseudocapsule (A, original magnification $\times 20$ ) with prominant lymphocyte infiltration into small vessel wall (arrow) (B, original magnification $\times 200)$. Immunohistochemistry stains revealed positive for CD3 (C) and CD31 (D), and these markers are related to lymphocytes (original magnification $\times 200$ ).

mating outcome and prognosis. However, the radiologic findings of PCNSV may be nonspecific; multifocal, leptomeningeal dissemination or high-grade tumors, including metastatic brain tumors or glioma, have been reported [3]. Furthermore, routine MRI is sometimes negative, even when primary or secondary vasculitis is confirmed [4-6]. Thus, the diagnosis of PCNSV based on radiologic findings can be unreliable.

Recent reports suggest that MR spectroscopy is useful in the diagnosis of PCNSV, as PCNSV had no significant elevation of the choline peak, which one would expect with an aggres- sive brain tumor. The most remarkable abnormality in PCNSV might be marked elevation of the glutamate and glutamine peaks, which is a kind of neurotransmitter primarily found in astrocytes. In inflammatory conditions, cell breakdown of neural and glial elements occurs, along with an associated adjacent astrocytic response, which leads to a local accumulation of many metabolites, including a high concentration of glutamine and glutamate [3,7]. Our patient had the typical findings of a high-grade malignant cystic brain tumor on conventional MRI; if his condition had permitted MR spectroscopy, that 
procedure may have made the diagnosis of vasculitis more likely.

Histologically, CNS vasculitis can be categorized into 3 types: granulomatous, lymphocytic, and necrotizing [8]. Granulomatous vasculitis is the most common type and has vasculocentric mononuclear and granulomatous inflammation. Lymphocytic vasculitis, the second most common type, has prominent lymphocytic inflammation associated with plasma cell infiltration and destruction of vessels. Necrotizing vasculitis, the least common type, is associated with transmural fibrinoid necrosis, which occasionally causes intracranial hemorrhage [9]. The present case had a cystic mass-like lesion, and the cyst capsule had prominent lymphocytic inflammation into vessel walls and destruction of vessels.

Regimens for treating cerebral vasculitis, such as with corticosteroid and immunosuppressant, are derived from strategies used for other types of vasculitis. Brain edema responds especially well to corticosteroids. Early corticosteroid treatment of PCNSV can often have favorable outcomes and prevent serious outcomes [10]. To our knowledge, despite the early administration of high-dose corticosteroids, rapid progression of the disease within a few days is very extremely rare. The reason our patient's disease progressed is not known. Nevertheless, the case suggests that the presence of a large mass-like brain lesion with severe edema should prompt efforts to make a tissue diagnosis promptly, so corticosteroid/ immunosuppressive therapy can be instituted if indicated.

In conclusion, an unusual case of cystic vasculitis with rapid progression despite early administration of corticosteroid therapy is presented. If malignant cystic tumor combined with severe brain edema is suspected, early biopsy or resection should be considered to confirm the diagnosis and permit early institution of appropriated treatments.

\section{Conflicts of Interest}

The authors have no potential conflicts of interest.

\section{Acknowledgments}

We also appreciate Wade Martin of Emareye for his critical English revision. This work was supported by the National Research Foundation of Korea (NRF) grant funded by the Korea government (Ministry of Science and ICT) (NRF-2018R1C1B5085134).

\section{REFERENCES}

1. Salvarani C, Brown RD Jr, Calamia KT, et al. Primary central nervous system vasculitis: analysis of 101 patients. Ann Neurol 2007;62:442-51.

2. MacLaren K, Gillespie J, Shrestha S, Neary D, Ballardie FW. Primary angiitis of the central nervous system: emerging variants. QJM 2005;98:643-54.

3. Panchal NJ, Niku S, Imbesi SG. Lymphocytic vasculitis mimicking aggressive multifocal cerebral neoplasm: MR imaging and MR spectroscopic appearance. AJNR Am J Neuroradiol 2005;26:642-5.

4. Imbesi SG. Diffuse cerebral vasculitis with normal results on brain MR imaging. AJR Am J Roentgenol 1999;173:1494-6.

5. Negishi C, Sze G. Vasculitis presenting as primary leptomeningeal enhancement with minimal parenchymal findings. AJNR Am J Neuroradiol 1993;14:26-8.

6. Shoemaker EI, Lin ZS, Rae-Grant AD, Little B. Primary angiitis of the central nervous system: unusual MR appearance. AJNR Am J Neuroradiol 1994;15:331-4.

7. Danielsen ER, Ross B. The clinical significance of metabolites. In: Magnetic resonance spectroscopy diagnosis of neurological diseases. New York: Marcel Dekker; 1999. p. 23-43.

8. Salvarani C, Brown RD Jr, Hunder GG. Adult primary central nervous system vasculitis. Lancet 2012;380:767-77.

9. Lee JS, Jung TY, Lee KH, Kim SK. Primary central nervous system vasculitis mimicking a cortical brain tumor: a case report. Brain Tumor Res Treat 2017;5:30-3.

10. Honda M, Koga M, Kanda T. [Treatment for central nervous system vasculitis]. Brain Nerve 2015;67:287-93. 